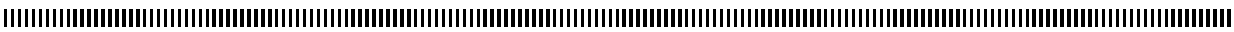

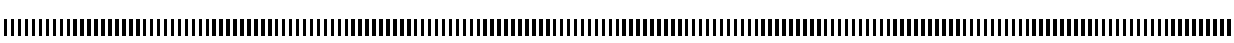

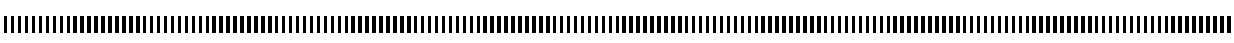

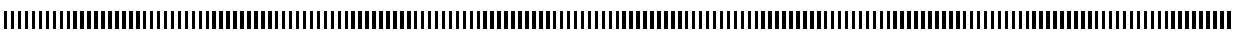

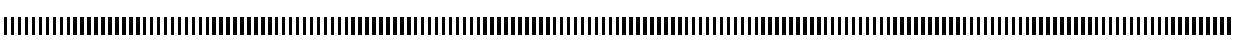

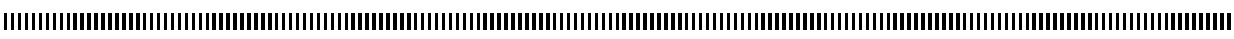

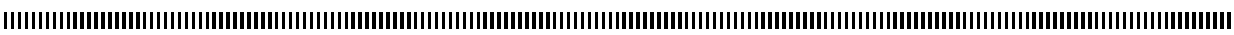

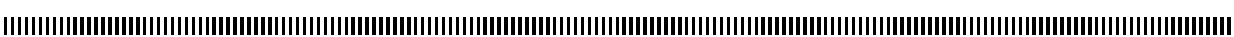

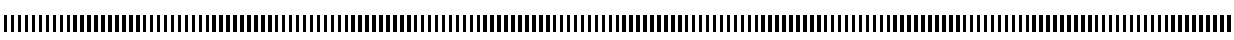

TAMTAM’09

\title{
Inverse impedance boundary problem via the conformal mapping method: the case of small impedances
}

\author{
F. Ben Hassen 1 , Y. Boukari ${ }^{1}$, H. Haddar ${ }^{2}$ \\ ${ }^{1}$ ENIT-LAMSIN, Ecole Nationale des Ingénieurs de Tunis BP 37, 1002 Tunis, Tunisia \\ fahmi.benhassen@enit.rnu.tn,yosra.boukari@lamsin.rnu.tn. \\ 2 INRIA Saclay Ile de France \& CMAP, Ecole Polytechnique, Route de Saclay, 91128 Palaiseau \\ Cedex, France \\ Houssem.Haddar@inria.fr
}

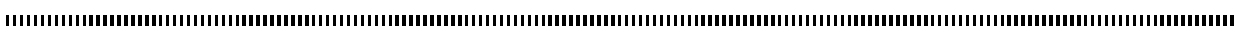

RÉSUMÉ. Haddar and Kress [9] ont étendu l'utilisation de la méthode des applications conformes $[2,8]$ pour construire le bord intérieur $\Gamma_{i}$ d'un domaine doublement connexe à partir de données de Cauchy sur le bord extérieur d'une fonction harmonique qui satisfait une condition homogène d'impédance sur $\Gamma_{i}$. Cependant, l'analyse de la méthode montre la non-convergence de l'algorithme proposé dans [9] dans le cas des faibles impédances. Dans ce travail, nous proposons des modifications de cet algorithme afin d'obtenir un schéma d'inversion convergent et stable dans ce cas. La méthode est ensuite validée par des exemples numériques qui incluent également le cas d'impédances variables.

ABSTRACT. Haddar and Kress [9] extended the use of the conformal mapping approach [2, 8] to reconstruct the internal boundary curve $\Gamma_{i}$ of a doubly connected domain from the Cauchy data on the external boundary of a harmonic function satisfying a homogeneous impedance boundary condition on $\Gamma_{i}$. However, the analysis of this scheme indicates non convergence of the proposed algorithm for small values of the impedance. In this paper, we modify the algorithm proposed in [9] in order to obtain a convergent and stable inversion process for small impedances. We illustrate the performance of the method through some numerical examples that also include the cases of variable impedances.

MOTS-CLÉS : tomographie électrique, problèmes inverses, applications conformes.

KEYWORDS : electric impedance tomography, inverse problems, conformal mapping.

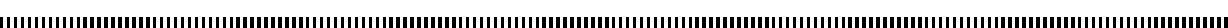

Reçu le 06/10/2009,

Revue ARIMA, vol. 13 (2010), pp. 47-62

révisé le 06/05/2010,

accepté le 13/09/2010 


\section{Introduction}

We are interested in an inverse problem for harmonic functions that arises in the mathematical modeling of electrostatic or thermal imaging methods. As examples of applications we can mention the detection of faults in metal plates via applying electric currents $[3,4]$, the monitoring of lung patients [7] or also the evaluation of wood quality both via electrical impedance tomography in long cylindrical objects [17]. In these applications, an unknown inclusion within a conducting host medium $D$ with constant conductivity is assessed from overdetermined Cauchy data on the accessible part of the boundary $\Gamma_{e}$ of the medium. We shall consider the cases where the effect of the inclusion on imposed currents can be modeled by an impedance boundary condition that has be to satisfied on its boundary $\Gamma_{i}$. This model can be viewed for instance as an approximation of the transmission problem in the asymptotic regimes : inclusion with a very high or a very small conductivity.

In a series of papers, a simple and fast numerical scheme was developed to reconstruct a perfectly conducting inclusion $[2,14]$ or a nonconducting inclusion [8], governed by a homogeneous Dirichlet or Neumann boundary condition on the boundary $\Gamma_{i}$ of the inclusion. The approach is based on a conformal mapping $\Psi: B \rightarrow D$ that takes an annulus $B$ bounded by two concentric circles onto $D$. In the first step, in terms of the given Cauchy data on $\Gamma_{e}$, by successive approximations one has to solve a non-local and nonlinear ordinary differential equation for the boundary values $\Psi_{\mid C_{1}}$ of this mapping on the exterior boundary circle of $B$. Then in the second step, a Cauchy problem for the holomorphic function $\Psi$ in $B$ has to be solved via a regularized Laurent expansion to obtain the unknown boundary $\Gamma_{i}=\Psi\left(C_{\rho}\right)$ as the image of the interior boundary circle $C_{\rho}$.

The application of the conformal mapping method to the inverse impedance problem was first analyzed in [9]. Since the homogeneous impedance condition on $\Gamma_{i}$ transforms into an impedance condition on $C_{\rho}$ that contains the trace of the derivative of the conformal map $\Psi$ on $C_{\rho}$, the method does not completely separate the two steps indicated above. The analysis of the proposed algorithm indicated the convergence for large impedances (i.e. when the impedance boundary condition is close to a Dirichlet boundary condition). However, as expected from known results on the Neumann problem, (and as numerically observed) the algorithm does not converge in the opposite regime, i.e. for small impedances. This paper is devoted to addressing this issue using similar ideas as in the treatment of the Neumann problem, namely by deriving the nonlinear equation of step 1) associated with the conjugate harmonic function. The impedance condition is transformed into a generalized impedance condition (where second order tangential derivative is involved) and therefore the subsequent analysis requires a special care. The analysis of the algorithm indicates convergence for sufficiently small impedances. This is confirmed numerically even in the case of variable impedances. As we shall point out theoretically, the values of the impedances for which convergence occurs depends on the size of the inclusion. This is also true in the case of [9]. For other work using conformal mapping ideas in the study of inverse problems for the Laplace equation we refer to [1, 5, 6, 12, 11]. Concerning the recovery of obstacles with impedance boundary conditions we also refer to $[15,16]$ where a Newton like method based on the use of appropriate integral representation of the solution is used. Compared to our method, this technique is indeed more general and allows one to seek both the impedance and the geometry of the obstacle. However, no convergence proof is available for this technique and as opposed to our algorithm, close enough initialization is required to ensure convergence. 
The paper is organized as follows. We introduce in Section 2 the inverse impedance problem and recall the general ideas of the conformal mapping method and the reconstruction process proposed in [9]. In section 3, we describe the modified algorithm for small values of the impedance. The convergence of the proposed algorithm is discussed in Section 4. Finally, we conclude in section 5 with some numerical implementations to valid our approach both for a constant and variable impedances.

\section{The inverse problem and the conformal mapping method}

Let $D$ be a doubly connected bounded domain in $\mathbf{R}^{2}$ with a smooth boundary $\partial D$ that consists of two disjoint closed Jordan curves $\Gamma_{i}$ and $\Gamma_{d}$, that is $\partial D=\Gamma_{i} \cup \Gamma_{e}$ with $\Gamma_{i} \cap \Gamma_{e}=\emptyset$ such that $\Gamma_{i}$ is contained in the interior of $\Gamma_{e}$. By $\nu$ we denote the outward unit normal to $\Gamma_{e}$. For a given function $f \in H^{1 / 2}\left(\Gamma_{e}\right)$ and a positive constant $\lambda$ we consider the impedance problem for the Laplace equation

$$
\Delta u=0 \quad \text { in } D,
$$

with boundary conditions

$$
\frac{\partial u}{\partial \nu}-\lambda u=0 \quad \text { on } \Gamma_{i}
$$

and

$$
u=f \quad \text { on } \Gamma_{e} .
$$

Note that the positivity of $\lambda$ ensures existence and uniqueness of a solution $u \in H^{1}(D)$.

The inverse problem we are interested in, is to determine the shape of the interior boundary curve $\Gamma_{i}$ from one or many pairs of Cauchy data

$$
(f, g)=\left.\left(u, \frac{\partial u}{\partial \nu}\right)\right|_{\Gamma_{e}},
$$

assuming that the impedance is known a priori. We shall use for that purpose the conformal mapping method introduced in $[2,8]$. We shall first describe the principle of this method and the setting used in [9] to solve the case of large values of $\lambda$.

Principle of the conformal mapping method : Let $B:=\{z \in \mathbf{C}: \rho<|z|<1\}$ denotes the annulus bounded by the two circles $C_{\rho}:=\{z \in \mathbf{C}:|z|=\rho\}$ and $C_{1}:=$ $\{z \in \mathbf{C}:|z|=1\}$. Since $D$ is a doubly connected domain, then by using the conformal mapping theorem (see [11]), there exists a uniquely determined radius $\rho$ and a bijective holomorphic function $\Psi: B \rightarrow D$ that conformally maps $B$ onto $D$ such that the boundaries $C_{\rho}$ and $C_{1}$ are mapped onto $\Gamma_{i}$ and $\Gamma_{e}$, respectively. Denoting by $L_{1}$ the length of $\Gamma_{e}$, and let $\gamma:\left[0, L_{1}\right] \rightarrow \Gamma_{e}$ be a parametrization of $\Gamma_{e}$ in terms of the arc length. The mapping $\Psi$ is uniquely characterized by prescribing $\Psi(1)=\gamma(0)$.

The inversion algorithm is based on the determination of $\rho$ and $\Psi$. To this end, we introduce the two functions : $\chi:[0,2 \pi] \rightarrow \mathbf{C}$ and $\varphi:[0,2 \pi] \rightarrow\left[0, L_{1}\right]$ defined by

$$
\chi(t):=\Psi\left(\rho e^{i t}\right) \quad \text { and } \quad \varphi(t):=\gamma^{-1}\left(\Psi\left(e^{i t}\right)\right) .
$$

The function $\chi$ is injective and parametrizes the interior boundary curve $\Gamma_{i}$. The function $\varphi$ is strictly monotonically increasing and bijective, and roughly speaking it describes how $\Psi$ maps arc length on $C_{1}$ onto arc length on $\Gamma_{e}$. Obviously, determining the map $\chi$ solves 
the inverse problem. The main idea behind setting up the conformal mapping method is the derivation of a non linear differential equation satisfied by the boundary map $\varphi$. This equation is then solved iteratively to determine $\varphi$ then obtain $\chi$.

Iterative operator for large impedances (see [9]) : The derivation of the non linear differential equation used in [9] for large impedances is done as follows. We associate the impedance problem (1)-(3) in $D$ with an impedance problem posed in the annulus $B$. More precisely, if $u$ solves the impedance problem (1)-(3) then $v:=u \circ \Psi$ is a harmonic function

$$
\Delta v=0 \text { in } B
$$

satisfying the boundary conditions

$$
\frac{\partial v}{\partial r}\left(\rho e^{i t}\right)-\mu_{\rho, \Psi}\left(\rho e^{i t}\right) v\left(\rho e^{i t}\right)=0, \quad 0 \leq t \leq 2 \pi
$$

and

$$
v=f \circ \Psi \quad \text { on } C_{1},
$$

where

$$
\mu_{\rho, \Psi}\left(\rho e^{i t}\right):=\frac{\lambda}{\rho}\left|\frac{d}{d t} \Psi\left(\rho e^{i t}\right)\right|=\frac{\lambda}{\rho}\left|\chi^{\prime}(t)\right|, \quad 0 \leq t \leq 2 \pi .
$$

Denote by $A_{\rho, \mu}: H^{1 / 2}\left(C_{1}\right) \rightarrow H^{-1 / 2}\left(C_{1}\right)$ the Dirichlet-to-Neumann operator for the impedance problem (6)-(8). It maps functions $f \circ \Psi \in H^{1 / 2}\left(C_{1}\right)$ onto the normal derivative $\partial v / \partial \nu$ on $C_{1}$ of the solution of (6)-(8).

Using the Cauchy-Riemann equations, we get the following non-local differential equation

$$
\frac{\partial \varphi}{\partial t}=\frac{A_{\rho, \mu}(f \circ \gamma \circ \varphi)}{g \circ \gamma \circ \varphi}
$$

for the boundary mapping $\varphi$ which has to be complemented by the boundary conditions

$$
\varphi(0)=0 \text { and } \varphi(2 \pi)=L_{1} .
$$

In order to rewrite (10)-(11) as a fixed point equation we introduce the affine operator $V$ :

$$
(V \psi)(t)=\frac{L_{1}}{2 \pi} t+\psi(t), \quad t \in[0,2 \pi] .
$$

Then, for a pair of Cauchy data $(f, g)$, after setting

$$
U_{\rho, \mu} \psi:=\frac{A_{\rho, \mu}(f \circ \gamma \circ V \psi)}{g \circ \gamma \circ V \psi},
$$

we define an operator $T_{\rho, \mu}: H_{0}^{1}[0,2 \pi] \rightarrow H_{0}^{1}[0,2 \pi]$ by

$$
\left(T_{\rho, \mu} \psi\right)(t):=\int_{0}^{t}\left[\left(U_{\rho, \mu} \psi\right)(\tau)-\frac{1}{2 \pi} \int_{0}^{2 \pi}\left(U_{\rho, \mu} \psi\right)(\theta) d \theta\right] d \tau, \quad t \in[0,2 \pi]
$$

Finally, if $\varphi \in H^{1}[0,2 \pi]$ is a solution of (10)-(11) then $\psi=V^{-1} \varphi \in H_{0}^{1}[0,2 \pi]$ is a fixed point of $T_{\rho, \mu}$.

The scheme proposed in [9] is mainly based on solving the fix point equation $T_{\rho, \mu} \psi=$ $\psi$ using successive approximations. However, in order to completely describe the scheme and obtain a convergent one we omitted three steps 1) stabilization of the division by $g$ in 
(10) by combining the use of two Cauchy data 2) derivation of an equation for the radius 3) regularization of the mapping $\varphi \rightarrow \chi$. One obtains a convergent algorithm for large impedances mainly because, as $\lambda \rightarrow \infty$ the operator $A_{\rho, \mu}$ "converges" to the DtN operator associated with a homogeneous Dirichlet boundary condition on the interior circle. One can also show that the algorithm is non convergent for sufficiently small impedances since in that case $A_{\rho, \mu}$ is close to the DtN map associated with a homogeneous Neumann boundary condition on the interior circle. To obtain a convergent scheme in the latter cases, we shall modify the right hand side of (10) using a DtN map associated with the conjugate harmonic. As $\lambda \rightarrow 0$ this operator has a similar behavior as $A_{\rho, \mu}$ when $\lambda \rightarrow \infty$.

\section{The new scheme for small impedances}

If $u$ is a harmonic function in $D$ with Cauchy data $(f, g)$ on $\Gamma_{e}$ satisfying the impedance condition (3) then from the Cauchy-Riemann equations it follows that a conjugate harmonic $\tilde{u}$ of $u$ satisfies

$$
\Delta \tilde{u}=0 \text { in } D
$$

with the "generalized" impedance condition

$$
\frac{\partial \tilde{u}}{\partial \nu}+\frac{1}{\lambda} \frac{\partial^{2} \tilde{u}}{\partial s^{2}}=0 \quad \text { on } \Gamma_{i}
$$

and the Cauchy data

$$
\begin{array}{r}
\frac{\partial \tilde{u}}{\partial s}=g \quad \text { on } \Gamma_{e} \\
\frac{\partial \tilde{u}}{\partial \nu}=-\frac{\partial f}{\partial s} \quad \text { on } \Gamma_{e} .
\end{array}
$$

We observe in particular, from integrating (16) over $\Gamma_{e}$, that the existence of single valued conjugate harmonic function $\tilde{u}$ is subjected to the condition

$$
\int_{\Gamma_{e}} g d s=0
$$

Let us assume for the moment that this condition is verified and let $\tilde{u}$ be the (unique) solution to (14)-(16). The $\tilde{v}:=\tilde{u} \circ \Psi$ satisfies

$$
\Delta \tilde{v}=0 \quad \text { in } B
$$

with the following generalized impedance boundary condition on $C_{\rho}$

$$
\frac{\partial \tilde{v}}{\partial r}\left(\rho e^{i t}\right)+\frac{1}{\rho^{2}} \frac{\partial}{\partial t}\left(\tilde{\mu}(t) \frac{\partial}{\partial t} \tilde{v}\left(\rho e^{i t}\right)\right)=0 \quad t \in[0,2 \pi]
$$

and the Dirichlet boundary condition on $C_{1}$

$$
\tilde{v}\left(e^{i t}\right)=\tilde{F}\left(e^{i t}\right) \quad t \in[0,2 \pi],
$$

where we have set :

$$
\tilde{\mu}(t):=\frac{\rho}{\lambda\left|\chi^{\prime}(t)\right|} \quad \text { and } \quad \tilde{F}\left(e^{i t}\right):=\tilde{f} \circ \varphi(t), \quad t \in[0,2 \pi],
$$


with $\tilde{f}$ denoting the function defined with respect to the arc length on $\Gamma_{e}$ by

$$
\tilde{f}(s)=\int_{0}^{s} g \circ \gamma(t) d t, \quad s \in\left[0, L_{1}\right] .
$$

Let $\tilde{A}_{\rho, \tilde{\mu}}: H^{1 / 2}\left(C_{1}\right) \rightarrow H^{-1 / 2}\left(C_{1}\right)$ be the Dirichlet-Neumann operator : $\tilde{F} \mapsto \partial \tilde{v} /\left.\partial_{r}\right|_{C_{1}}$, associated to the impedance problem (19)-(21). Similarly to above, using the CauchyRiemann equations, one get the following non-local differential equation

$$
\frac{\partial \varphi}{\partial t}=\frac{\tilde{A}_{\rho, \tilde{\mu}}(\tilde{f} \circ \varphi)}{\tilde{g} \circ \varphi}
$$

for the boundary mapping $\varphi$, which are complemented by the boundary conditions

$$
\varphi(0)=0 \text { and } \varphi(2 \pi)=L_{1} .
$$

The function $\tilde{f}$ is given by (23) whereas $\tilde{g}$ is defined in terms of the arc length on $\Gamma_{e}$ by

$$
\tilde{g}(s)=-(f \circ \gamma(s))^{\prime}, \quad s \in\left[0, L_{1}\right] .
$$

\subsection{Combination of three Cauchy pairs}

In order to get a stable reconstruction algorithm, the function $\tilde{g}$ should have no zeros. Moreover, the assumption (18) is not satisfied in general for some Dirichlet data $f$. To remedy to these problems, we use three different pairs of Cauchy data $\left(f_{i}, g_{i}\right), 1 \leq i \leq 3$. We assume that at least one of the three pairs does not satisfy (18), for example the pair number 3 . We then build two pairs of Cauchy data $\left(\hat{f}_{i}, \hat{g}_{i}\right), i=1$, 2 , verifying (18) by setting :

$$
\hat{f}_{i}:=f_{i}-\theta_{i} f_{3} \text { and } \hat{g}_{i}:=g_{i}-\theta_{i} g_{3} .
$$

where

$$
\theta_{i}=\left(\int_{\Gamma_{e}} g_{i} d s\right) /\left(\int_{\Gamma_{e}} g_{3} d s\right)
$$

We define $\tilde{f}_{i}$ and $\tilde{g}_{i}$ as in (23) and (26) where $f$ and $g$ are respectively replaced with $\hat{f}_{i}$ and $\hat{g}_{i}$. It is then clear from 24 that

$$
\frac{\partial \varphi}{\partial t}=\frac{\tilde{A}_{\rho, \tilde{\mu}}\left(\tilde{f}_{i} \circ \varphi\right)}{\tilde{g}_{i} \circ \varphi}=\frac{\left(\tilde{g}_{i} \circ \varphi\right) \tilde{A}_{\rho, \tilde{\mu}}\left(\tilde{f}_{i} \circ \varphi\right)}{\left|\tilde{g}_{i} \circ \varphi\right|^{2}}
$$

for $i=1,2$. To shorten the notation, we introduce the complex-valued functions (which have therefore different meaning from those introduced above)

$$
\tilde{f}=\tilde{f}_{1}+i \tilde{f}_{2} \quad \text { and } \quad \tilde{g}=\tilde{g}_{1}+i \tilde{g}_{2}
$$

and observe that (27) implies that one also have

$$
\frac{d \varphi}{d t}=\frac{\Re\left\{(\tilde{g} \circ \varphi) \tilde{A}_{\rho, \tilde{\mu}}(\tilde{f} \circ \varphi)\right\}}{|\tilde{g} \circ \varphi|^{2}} .
$$

In order to write (28) as a fixed point equation, we define the operator $T_{\rho, \tilde{\mu}}^{\text {mod }}: H_{0}^{1}[0,2 \pi] \rightarrow$ $H_{0}^{1}[0,2 \pi]$ by

$$
\left(T_{\rho, \tilde{\mu}}^{m o d} \psi\right)(t):=\int_{0}^{t}\left[\left(U_{\rho, \tilde{\mu}}^{m o d} \psi\right)(\tau)-\frac{1}{2 \pi} \int_{0}^{2 \pi}\left(U_{\rho, \tilde{\mu}}^{m o d} \psi\right)(\theta) d \theta\right] d \tau, \quad t \in[0,2 \pi] .
$$


where

$$
U_{\rho, \tilde{\mu}}^{m o d} \psi:=\frac{\Re\left\{(\tilde{g} V \psi) \tilde{A}_{\rho, \tilde{\mu}}(\tilde{f} V \psi)\right\}}{|\tilde{g} V \psi|^{2}} .
$$

Here, the operator $V$ is still given by (12). Obviously, if $\varphi \in H^{1}[0,2 \pi]$ is a solution of (28) satisfying (25) then $\psi=V^{-1} \varphi \in H_{0}^{1}[0,2 \pi]$ is a fixed point of $T_{\rho, \tilde{\mu}}^{\text {mod }}$, that is

$$
\psi=T_{\rho, \tilde{\mu}}^{\bmod }(\psi)
$$

is satisfied. Note that if $T_{\rho, \tilde{\mu}}^{\text {mod }}$ is a contraction, then (28)-(25) and (30) are equivalent.

\subsection{Equations for $\rho$}

The fixed point equation (30) has to be coupled with an equation for the unknown radius $\rho$. We shall adopt a similar procedure as in [9]. Let $w$ be a harmonic function in $B$ satisfying the generalized impedance boundary condition (21) on $C_{\rho}$, with $\tilde{\mu}$ as defined by (22). Moreover, we assume that $w=F_{1}$ on $C_{1}$ for a given $F_{1} \in H^{1 / 2}\left(C_{1}\right)$. Then, we introduce the function $H_{\Psi}:(0,1) \rightarrow \mathbf{R}$ given by

$$
H_{\Psi}(\rho):=\int_{C_{1}} \tilde{f} \circ \varphi \frac{\partial w}{\partial \nu} d s .
$$

Applying the Green's theorem to $\tilde{v}=\tilde{u} \circ \Psi$ and $w$ in the annulus $B$, and since the two functions satisfy the same generalized impedance condition on the interior boundary $C_{\rho}$,

$$
\int_{C_{1}} \tilde{v} \frac{\partial w}{\partial \nu} d s=\int_{C_{1}} \frac{\partial \tilde{v}}{\partial \nu} F_{1} d s=\int_{0}^{2 \pi} \tilde{g}(\gamma(\varphi(t))) \varphi^{\prime}(t) F_{1}(t) d t
$$

Therefore, we have

$$
H_{\Psi}(\rho)=\int_{0}^{2 \pi} \tilde{g}(\gamma(\varphi(t))) \varphi^{\prime}(t) F_{1}(t) d t
$$

To compute an approximate solution of (32) via Newton iterations, we need the derivative of $H_{\Psi}$. This in turn requires to calculate the derivative $w^{\prime}$ of the function $w$.

$w^{\prime}$ is a harmonic function in $B$ vanishing on $C_{1}$. To derive the boundary condition satisfied by $w^{\prime}$ on $C_{\rho}$, we indicate the dependence of $w$ on the radius $\rho$ by writing $w_{\rho}$ and expand,

$$
\begin{aligned}
\frac{\partial w_{\rho+\delta}}{\partial r}(\rho+\delta)+\frac{1}{(\rho+\delta)^{2}} \frac{\partial}{\partial t}\left(\tilde{\mu}_{\rho+\delta} \frac{\partial w_{\rho+\delta}}{\partial t}\right)(\rho+\delta) \\
=\frac{\partial w_{\rho}}{\partial r}(\rho)+\frac{1}{\rho^{2}} \frac{\partial}{\partial t}\left(\tilde{\mu}_{\rho} \frac{\partial w_{\rho}}{\partial t}\right)(\rho) \\
\quad+\frac{1}{\rho^{2}}\left\{\frac{\partial}{\partial t}\left(\tilde{\mu}_{\rho} \frac{\partial w_{\rho}^{\prime}}{\partial t}\right)(\rho)+\frac{\partial}{\partial t}\left(\tilde{\mu}_{\rho}^{\prime} \frac{\partial w_{\rho}}{\partial t}\right)(\rho)+\frac{\partial}{\partial t}\left(\tilde{\mu}_{\rho} \frac{\partial}{\partial t} \frac{\partial w_{\rho}}{\partial r}\right)(\rho)\right\} \delta \\
+\left\{\frac{\partial w_{\rho}^{\prime}}{\partial r}(\rho)+\frac{\partial^{2} w_{\rho}}{\partial r^{2}}(\rho)-\frac{2}{\rho^{3}} \frac{\partial}{\partial t}\left(\tilde{\mu}_{\rho} \frac{\partial w_{\rho}}{\partial t}\right)(\rho)\right\} \delta+O\left(\delta^{2}\right)
\end{aligned}
$$


In view of the boundary condition (21) for $w_{\rho+\delta}$ and $w_{\rho}$ and after dropping the subscript $\rho$, we have

$$
\begin{aligned}
& \frac{\partial w^{\prime}}{\partial r}+\frac{\partial}{\partial s}\left(\tilde{\mu} \frac{\partial w^{\prime}}{\partial s}\right) \\
& =-\frac{\partial^{2} w}{\partial r^{2}}+\frac{2}{\rho} \frac{\partial}{\partial s}\left(\tilde{\mu} \frac{\partial w}{\partial s}\right)-\frac{\partial}{\partial s}\left(\tilde{\mu}^{\prime} \frac{\partial w}{\partial s}\right)-\frac{\partial}{\partial s}\left(\tilde{\mu} \frac{\partial}{\partial s} \frac{\partial w}{\partial r}\right) \quad \text { on } C_{\rho} .
\end{aligned}
$$

Therefore, using the Laplace operator in polar coordinates and once again the boundary condition (21) for $w$, the derivative $w^{\prime}$ satisfies on $C_{\rho}$

$$
\begin{aligned}
\frac{\partial w^{\prime}}{\partial r}+\frac{\partial}{\partial s}\left(\tilde{\mu} \frac{\partial w^{\prime}}{\partial s}\right)= & \frac{1}{\rho} \frac{\partial w}{\partial r}+\frac{\partial^{2} w}{\partial s^{2}}+\frac{2}{\rho} \frac{\partial}{\partial s}\left(\tilde{\mu} \frac{\partial w}{\partial s}\right) \\
& -\frac{\partial}{\partial s}\left(\tilde{\mu}^{\prime} \frac{\partial w}{\partial s}\right)-\frac{\partial}{\partial s}\left(\tilde{\mu} \frac{\partial^{2}}{\partial s^{2}}\left(\tilde{\mu} \frac{\partial w}{\partial s}\right)\right)
\end{aligned}
$$

Finally, the derivative of $H_{\Psi}$ is

$$
H_{\Psi}^{\prime}(\rho)=\int_{C_{1}} \tilde{v} \frac{\partial w^{\prime}}{\partial \nu} d s
$$

where $w^{\prime}$ is a harmonic function in $B$ which vanishes on $C_{1}$ and satisfies the boundary condition 33 on $C_{\rho}$.

\subsection{Regularization of the mapping $\varphi \mapsto \chi$}

The nonlinear equations for $\phi$ and $\rho$ (which use in particular the impedance $\tilde{\mu}$ ) as well as the reconstruction of $\Gamma_{i}$ requires a (stable) determination of $\chi$ in terms of $\phi$ and $\rho$. More precisely, knowing the radius $\rho$ and $\Psi\left(e^{i t}\right)=(\gamma \circ V \psi)(t)$, we need to solve the Cauchy problem for determining the holomorphic function $\Psi$ from its values on $C_{1}$. Expanding in a Fourier series

$$
(\gamma \circ V \psi)(t)=e^{i t} \sum_{k=-\infty}^{\infty} a_{k} e^{i k t}, \quad t \in[0,2 \pi],
$$

we obtain $\Psi$ by the Laurent series

$$
\Psi(z)=z \sum_{k=-\infty}^{\infty} a_{k} z^{k} .
$$

The severe ill-posedness of the Cauchy problem inherited from the ill-posedness of our inverse problem is clearly illustrated, since small errors in the Fourier coefficients $a_{k}$, $k<0$, will be amplified by the exponentially increasing factors $\rho^{k}$. Hence, we incorporate a Tikhonov-type regularization and replace (35) by

$$
\Psi_{\alpha}(z)=z \sum_{k=0}^{\infty} a_{k} z^{k}+z \sum_{k=1}^{\infty} a_{-k} \frac{|z|^{2 k}}{\alpha+|z|^{2 k}} z^{-k}, \quad 0<|z|<1,
$$

where $\alpha>0$ serves as a regularization parameter. We then get $\chi$ via $\chi=$ For its choice via a discrepancy principle we refer to [8]. To simplify notation, in the sequel by $L_{\alpha}$ we denote the map that takes $\psi$ into $\Psi_{\alpha}$ as defined by (36). 


\subsection{The inversion scheme}

Now, we can summarize our inversion process in the following iteration scheme. Given an initialization $\psi_{0}=0$ (see Remark 3.1) for the boundary map and an arbitrary choice for the initial radius by $\rho_{0}$ (we used $\rho_{0}=0.3$ in the numerical examples), the iteration algorithm is slitted into the following steps

1) Compute the mapping $\Psi_{\alpha}=L_{\alpha} \psi_{n}$ and the corresponding $\chi(t)=\Psi_{\alpha}\left(\rho_{n} e^{i t}\right)$.

2) Compute $H_{\Psi_{\alpha}}$ given by (31) and its derivative, by evaluating $w$ and $w^{\prime}$ associated with a chosen function $F_{1}$ on the unit circle.

3) Update the radius using one Newton iteration via

$$
\rho_{n+1}=\rho_{n}-\frac{H_{\Psi_{\alpha}}\left(\rho_{n}\right)-\int_{0}^{2 \pi} \tilde{g}\left(\gamma\left(V \psi_{n}(t)\right)\right)\left(V \psi_{n}\right)^{\prime}(t) F_{1}(t) d t}{H_{\Psi_{\alpha}}^{\prime}\left(\rho_{n}\right)} .
$$

4) Update the impedance function $\tilde{\mu}_{n+1}=\frac{\rho_{n+1}}{\lambda\left|\chi^{\prime}(t)\right|}$.

5) Update the mapping $\varphi_{n+1}=V \psi_{n+1}$ by solving the differential equation associated with the iteration

$$
\psi_{n+1}=T_{\rho_{n+1}, \tilde{\mu}_{n+1}}^{\bmod }\left(\psi_{n}\right) .
$$

Remark 3.1 A natural initial guess for $\psi$ would be the one that corresponds with $\Psi$ being the conformal mapping that transforms the unit disk onto the domain enclosed by $\Gamma_{1}$. Since in our experiments we choose the to be the unit disk, this initial guess coincides with $\psi_{0}=0$.

\section{Convergence Analysis}

In order to check the convergence of our algorithm for small impedances we shall restrict ourselves to the simple case where the geometry is a small perturbation of an annulus. Moreover we shall assume that the exact value for the radius is known (and therefore, in particular $\tilde{\mu}=1 / \lambda)$. The analysis is then reduced to evaluating $\left\|\tilde{T}^{\prime}(0)\right\|$ where

$$
\tilde{T} \psi:=T_{\rho, 1 / \lambda}^{\bmod }(\psi)
$$

Following [8], one has

$$
\frac{d}{d t} \tilde{T}^{\prime}(0) h=\tilde{U}^{\prime}(0) h-\frac{1}{2 \pi} \int_{0}^{2 \pi} \tilde{U}^{\prime}(0) h d \tau .
$$

where

$$
\tilde{U}^{\prime}(0) h=\frac{\Re\left[\overline{\tilde{g}} \tilde{A}_{\rho, 1 / \lambda}\left(h \tilde{f}^{\prime}\right)-h \overline{\tilde{g}}^{\prime} \tilde{g}\right]}{|\tilde{g}|^{2}}
$$

We shall further restrict the analysis by choosing three pairs of Cauchy data $\left(f_{i}, g_{i}\right), 1 \leq$ $i \leq 3$, associated to

$$
f_{1}(\gamma(t))=\cos m t, \quad f_{2}(\gamma(t))=\sin m t, \quad f_{3}(\gamma(t))=1
$$

where $t \in[0,2 \pi]$ and $m \geq 1$. 
For abbreviation, by $c_{n}, s_{n}$ and $e_{n}$ we denote the trigonometric functions given by

$$
c_{n}(t):=\cos n t, \quad s_{n}(t):=\sin n t \quad \text { and } \quad e_{n}(t):=e^{i n t}
$$

for $n=0, \pm 1, \pm 2, \ldots$ Then one easily check that

$$
\tilde{A}_{\rho, 1 / \lambda} e_{n}=\gamma_{n} e_{n}
$$

with

$$
\gamma_{n}=|n| \frac{1-b_{n}}{1+b_{n}} \text { and } b_{n}=\frac{\lambda \rho-|n|}{\lambda \rho+|n|} \rho^{2|n|}
$$

while for $n \neq 0$,

$$
A_{\rho, \lambda} e_{n}=\tilde{\gamma}_{n} e_{n}
$$

with

$$
\tilde{\gamma}_{n}=|n| \frac{1+b_{n}}{1-b_{n}}
$$

We therefore conclude that

$$
\hat{g}_{1}(\gamma(t))=g_{1}(\gamma(t))=\tilde{\gamma}_{m} c_{m}(t), \text { and } \hat{g}_{2}\left(\gamma(t)=g_{2}(\gamma(t))=\tilde{\gamma}_{m} s_{m}(t) .\right.
$$

Consequently,

$$
\tilde{f}_{1}(t)=\frac{1}{m} \tilde{\gamma}_{m} s_{m}(t), \text { and } \hat{f}_{2}(t)=-\frac{1}{m} \tilde{\gamma}_{m} c_{m}(t)
$$

and

$$
\tilde{g}_{1}(t)=m s_{m}(t), \text { and } \hat{g}_{2}(t)=-m c_{m}(t) .
$$

After simple manipulations, and using $\tilde{\gamma}_{m} / m^{2}=1 / \gamma_{m}$, the expression of $U^{\prime}(0)$ simplifies into

$$
\tilde{U}^{\prime}(0) h=\frac{m}{\gamma_{m}} \Im\left(\bar{e}_{m} A_{\rho, 1 / \lambda}\left(h e_{m}\right)\right) .
$$

We are now in a situation similar to the convergence proof in [8, Theorem 3.1]. We conclude that

$$
\tilde{U}^{\prime}(0) s_{n}=\frac{m}{2 \gamma_{m}}\left[\gamma_{n+m}-\gamma_{n-m}\right] c_{n}
$$

and consequently, by integration,

$$
\tilde{T}^{\prime}(0) s_{n}=\frac{m\left[\gamma_{n+m}-\gamma_{n-m}\right]}{2 n \gamma_{m}} s_{n}
$$

for $n \in \mathbb{N}$. Analogously, $U^{\prime}(0)\left(c_{n}-1\right)=-\frac{m}{2 \gamma_{m}}\left[\gamma_{n+m}-\gamma_{n-m}\right] s_{n}$ and

$$
\tilde{T}^{\prime}(0)\left(c_{n}-1\right)=\frac{m\left[\gamma_{n+m}-\gamma_{n-m}\right]}{2 n \gamma_{m}}\left(c_{n}-1\right)
$$

for $n \in \underset{\mathbb{N}}{\mathbb{N}}$. We therefore deduce that the eigenvalues of the compact and self-adjoint operator $\tilde{T}^{\prime}(0)$ are

$$
\beta_{n}(\lambda, \rho)=\frac{m\left[\gamma_{n+m}-\gamma_{n-m}\right]}{2 n \gamma_{m}}
$$


For $n>0$ and $\rho<1$, we notice that as $\lambda \rightarrow 0$

$$
\beta_{n}(\lambda, \rho) \rightarrow \beta_{n}(0, \rho)
$$

where $\beta_{n}(0, \rho)$ are the eigenvalues of the iterative operator in the case of an inclusion with a homogeneous Dirichlet boundary condition. Using the result of [8, Theorem 3.1] we conclude that $\sup _{n}\left|\beta_{n}(0, \rho)\right|<1$ and,

$$
\lim _{\lambda \rightarrow 0}\left\|\tilde{T}^{\prime}(0)\right\|<1 .
$$

We therefore can state the following theorem :

Theorem 4.1 Assume that $D=B$ and that the pairs of data correspond to (40). Then the sequence $\left(\psi_{n}\right)$ defined by the successive approximations $\psi_{n+1}=\tilde{T} \psi_{n}$ where $\tilde{T}$ is defined by (37) converges to 0 , provided that $\lambda$ is sufficiently small

In practice it is desirable to a quantification of the value of $\lambda$ for which convergence occur. This question was not studied in [9]. We shall indicate in the following that the values of $\lambda$ for which convergence holds depends on $\rho$. This means in particular that the convergence in (44) is not uniform with respect to $\rho$ for all $n$. To see this it is sufficient to consider the case of

$$
\beta_{m}(\lambda, \rho)=\frac{\gamma_{2 m}}{\left(2 \gamma_{m}\right)}=\frac{\left(1-b_{2 m}\right)\left(1+b_{m}\right)}{\left(1-b_{m}\right)\left(1+b_{2} m\right)} .
$$

For $\lambda>0$, we easily see that

$$
\lim _{\rho \rightarrow 1} \beta_{m}(\lambda, \rho) \rightarrow \beta_{m}(\lambda, 1)=2 .
$$

This implies for instance that for any value of $0<\lambda<m$ there exists a radius $\rho$ close to 1 such that $\left\|\tilde{T}^{\prime}(0)\right\|>1$ and therefore the algorithm is not converging for that radius.

We conclude this section by indicating that the "non convergence region" for small values of $\lambda$ is confined in a neighborhood of $\rho=1$ whose width exponentially goes to 0 as $\lambda \rightarrow 0$. Computing the derivative of $\gamma_{n}$ with respect to $n$ indicates that $n \rightarrow \gamma_{n}$ is decreasing for

$$
n^{2} \geq n_{0}^{2}:=(\lambda \rho)^{2}-(\lambda \rho) / \ln (\rho)
$$

Therefore, following the similar procedure as in the proof of [8, Theorem 3.1], one can prove :

Theorem 4.2 Let $\beta_{n}(\lambda, \rho)$ be defined by (43) for $\lambda>0$ and $0<\rho<1$ and let $n_{0}$ be defined by (46). Then

$$
0<\beta_{n}(\lambda, \rho)<1 \text { for all }\left\{m \geq 1 \text { and }|n-m| \geq n_{0}\right\} \text { or }\left\{n=m \text { and } n+m \geq n_{0}\right\} .
$$

In particular we see that if $n_{0} \leq 1$ then,

$$
0<\beta_{n}(\lambda, \rho)<1 \text { for all } n \geq 1 .
$$

For sufficiently small $\lambda, n_{0} \leq 1$ for all $0<\rho \leq \rho_{0}(\lambda)$ where $\rho_{0}$ satisfies

$$
\rho_{0}=\exp \left(-\left(\lambda \rho_{0}\right) /\left(1-\left(\lambda \rho_{0}\right)^{2}\right)\right) .
$$


One observes that $\rho_{0} \rightarrow 1$ as $\lambda \rightarrow 0$ exponentially fast.

We also remark that the order $n_{0}$ can be used to quantify the term "small impedance" for a given value of the radius $\rho$ : these small values should correspond with $n_{0} \leq 1$. For example for $\rho=0.3, n_{0}$ is smaller than 1 if the value of the impedance is smaller than 2.25 .

We finally notice that this non uniform behavior with respect to $\rho$ is not restricted to the case of small impedances. We expect that similar results hold in the case of high impedances.

\section{Numerical Results}

In this section, we provide some numerical examples to illustrate the convergence of our algorithm. In these examples, we consider the case $m=1$ (for the data (40)), the unit circle as the exterior boundary and different parametrization of the interior boundary. The simulation of the data and the evaluation of the DtN map in the iteration procedure is done after reformulating the boundary value problem into an integral equation on the boundary of the domain. The corresponding integral equation is discretized using a Nyström method [13]. For the case of a generalized impedance boundary condition (which is needed to evaluate $\tilde{T}$ ), the second order tangential derivative is discretized using a standard finite difference method.
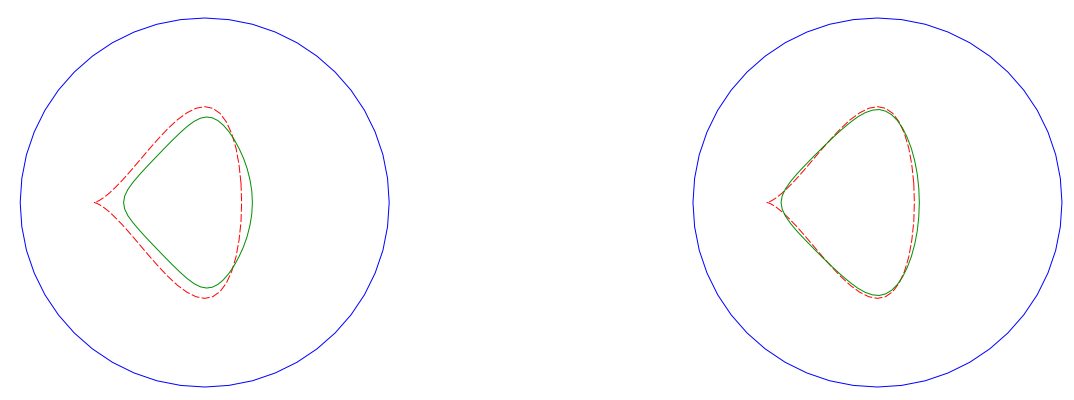

Figure 1. Exact (red) and reconstructed (green) geometries after 2 iterations (left) and 6 iterations (right). $\lambda=0.01$ and the Cauchy data to $f_{1}=\sin t, f_{2}=\cos t$ and $f_{3}=1$. No added random noise. 

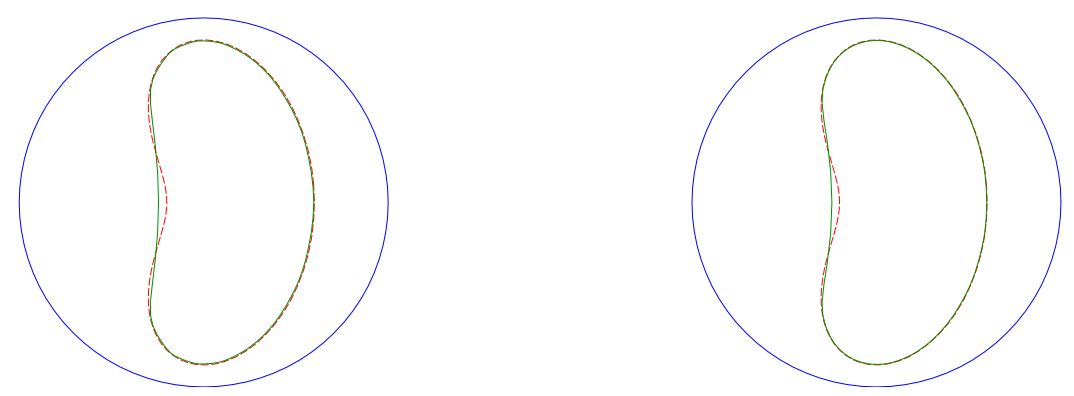

Figure 2. Exact (red) and reconstructed (green) geometries after 10 iterations (left) and 15 iterations (right). $\lambda=0.01$ and the Cauchy data to $f_{1}=\sin t, f_{2}=\cos t$ and $f_{3}=1$. No added random noise.

Figure 1 and Figure 2 respectively correspond to the two following parameterizations of the inetrior boundary $\Gamma_{i}$ :

$$
x_{1}(t)=-0.2+0.4 \cos t, \quad x_{2}(t)=0.4 \sin t+0.2 \sin t, t \in[0,2 \pi], \text { and }
$$

$$
x_{1}(t)=0.4 \cos t+0.2 \cos 2 t, \quad x_{2}(t)=0.8 \sin t+0.2 \sin 2 t, t \in[0,2 \pi] .
$$

As shown in Figure 1 and Figure 2, we find for $\lambda=0.01$ and after only 2 iterations a geometry close to the exact one.
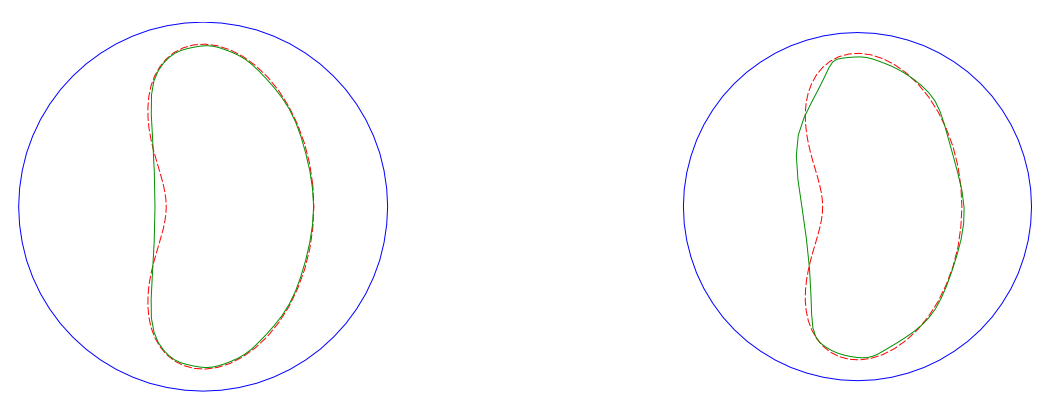

Figure 3. Exact (red) and reconstructed (green) geometries after 10 iterations for $\lambda=0.1$ and three Cauchy data corresponding to $f_{1}=\sin t, f_{2}=\cos t$ and $f_{3}=1$. Reconstruction done for different levels of added relative random noise : $1 \%$ (left), 10\% (right). 

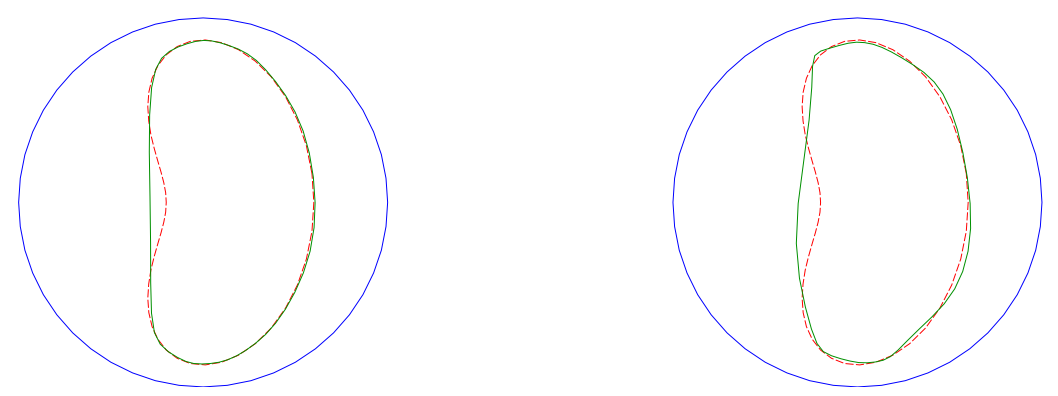

Figure 4. Exact (red) and reconstructed (green) geometries after 10 iterations for $\lambda=$ 0.001 and three Cauchy data corresponding to $f_{1}=\sin t, f_{2}=\cos t$ and $f_{3}=1$. Reconstruction done for different levels of added relative random noise : $1 \%$ (left), $10 \%$ (right).

As shown in Figure 3 and Figure 4, even with the presence of noise, we find a good reconstruction of the interior geometry.

The case of variable impedances In previous examples we considered the case of an inclusion with constant impedances. In some cases, this impedance would model the roughness of the inclusion boundary which implies that the impedance may be variable. Even though not supported by the convergence analysis, we shall numerically test our algorithm in the case of variable impedances. The only needed modification in our algorithm is to change the expression of the impedance $\tilde{\mu}$ into

$$
\tilde{\mu}(t)=\frac{1}{\lambda(\arg (\chi(t))} \frac{\rho}{\left|\chi^{\prime}(t)\right|}
$$

where arg denotes the argument of the complex number associated with a given vector.

We have tested the algorithm in the case $\lambda(t)=0.001(\sin (t)-1.001)$ for different geometries and obtain convergent and stable reconstructions as attested by Figures 5 and 6 .
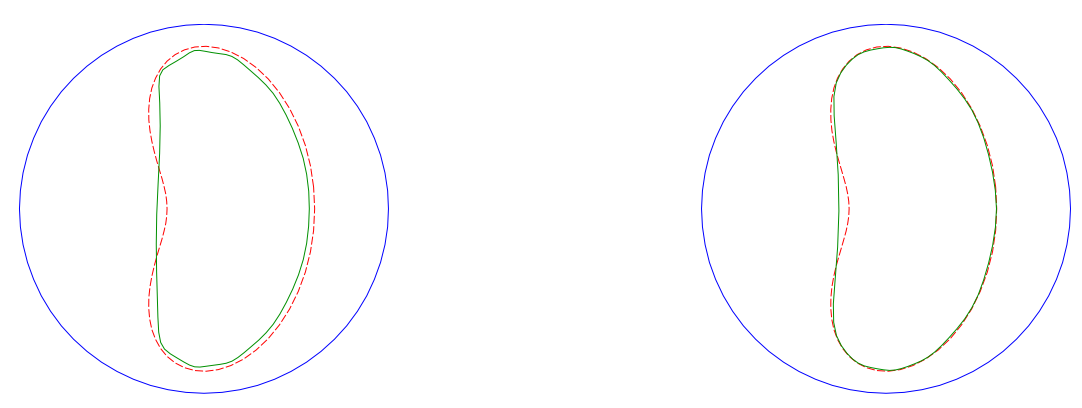

Figure 5. Exact (red) and reconstructed (green) geometries after 9 iterations (left) and 15 iterations (right) for $\lambda=0.001(\sin (t)-1.001)$ and three Cauchy data $f_{1}\left(e^{i t}\right)=$ $\cos t, f_{2}\left(e^{i t}\right)=\sin t$ and $f_{3}\left(e^{i t}\right)=1$. 

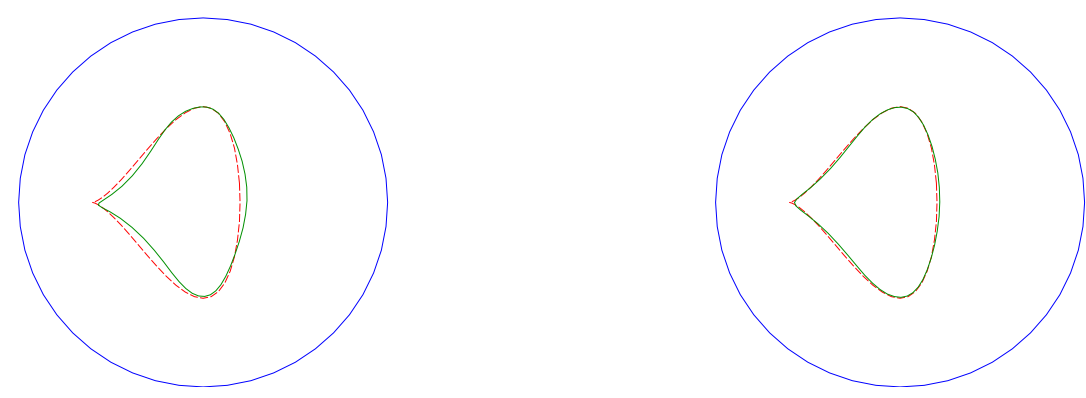

Figure 6. Exact (red) and reconstructed (green) geometries after 9 iterations (left) and 15 iterations (right) for $\lambda=0.001(\sin (t)-1.001)$ and three Cauchy data $f_{1}\left(e^{i t}\right)=$ $\cos t, f_{2}\left(e^{i t}\right)=\sin t$ and $f_{3}\left(e^{i t}\right)=1$.

\section{Conclusion}

We have studied the problem of determining the shape of a simply connected obstacle inside a simply connected domain, from the knowledge of Cauchy data associated with an electric potential, using the conformal mapping method. The potential satisfies the Laplace equation with an impedance boundary condition on the boundary of the obstacle. Our investigations focus on the case of small impedances as being an extension of the work proposed in [9]. We explained how a modification of the algorithm in [9] using conjugate harmonic functions provides a convergent iterative scheme. The convergence proof of the proposed algorithm is done in the simple case where the domain is a small perturbation of an annulus. Extending our approach to general geometries would be desirable, but is not obvious, since it is based on evaluating the spectral radius of the derivative of the iterative operator. The expression of this operator is very complex and hardly exploitable in the general case. We also pointed out, in the convergence analysis, that for a given value of $\lambda$, the algorithm is not convergent for all possible values of the radius $\rho$. We gave a precise quantification of this behavior for the case of an annulus. These theoretical considerations have been validated by means of some numerical tests. Those tests have been conducted for different configurations (that are not small perturbations of an annulus) and small impedance values, demonstrating the efficiency of the proposed algorithm. A perspective of the present work would the extension of this approach to the case of inclusions with small conductivities, which would be complementary to the work initiated in [10].

\section{Bibliographie}

[1] G. Alessandrini, A. Morassi, E. Rosset, « Detecting cavities by electrostatic boundary measurements. », Inverse Problems, vol. 18 , n 5 , 1333-1353, 2002.

[2] I. Akduman, R. KRess, " Electrostatic imaging via conformal mapping. », Inverse Problems, vol. $18, \mathrm{n}^{\circ} 6,2002$. 
[3] N. D. ApARicio, M. K. Pidcock, « The boundary inverse problem for the Laplace equation in two dimensions. », Inverse Problems, vol. 12, 565-77, 1996.

[4] H. T. BANKS, F. KoJIMA, « Boundary shape identification in two-dimensional electrostatic problems using SQUIDs. », J. Inv. Ill-Posed Problems vol. 8, 467-504, 2000.

[5] L. Baratchart, A. Ben Abda, F. Ben Hassen, J. Leblond, « Recovery of pointwise sources or small inclusions in 2D domains and rational approximation. », Inverse Problems vol. $21, \mathrm{n}^{\circ} 1,51-74,2005$.

[6] S. ChaAbane, C. Elhechmi, M. Jaoua, « A stable recovery method for the Robin inverse problem. », Math. Comput. Simulation vol. 66, n 4-5, 367-383, 2004.

[7] I. Frerichs, J. Hinz, P. Herrman, G. Weisser, G. Hahn, M. Quintel, G. Hellige. « Regional lung perfusion as determined by electrical impedance tomography in comparison with electron beam CT imaging. IEEE Trans. Med Imaging », vol. 21, 646-52, 2002.

[8] H. HADDAR, R. KRESS, « Electrostatic imaging via conformal mapping. », Inverse Problems, vol. $21, \mathrm{n}^{\mathrm{O}} 3,2005$.

[9] H. HAdDAR, R. KRESS, « Conformal mapping and an inverse impedance boundary value problem. », J. Inv. Ill-Posed Problems, vol. 14, $\mathrm{n}^{\mathrm{o}} 1,2006$.

[10] H. Haddar, R. Kress, " Conformal mapping and impedance tomography. », Inverse Problems, vol. 26, $\mathrm{n}^{\circ}$ 7, 2010.

[11] P. HenriCI « Applied and computational complex analysis », vol. 1, Wiley Classics Library, New York, 1988.

[12] M. JaOua, J. Leblond, M. MAhjoub, J. R. ,PARTington, « Robust numerical algorithms based on analytic approximation for the solution of inverse problems in annular domains. ", IMA J. Appl. Math, vol. 74 , n ${ }^{\circ}$ 2, 481-506, 2009.

[13] R. KRESS. Linear integral equations, volume 82 of Applied Mathematical Sciences. SpringerVerlag, New York, second edition, 1999.

[14] R. KRESS. « Inverse Dirichlet problem and conformal mapping », Math. and Computer in Sim. vol. 66, 255-65, 2004.

[15] W. RUNDELL « Recovering an obstacle and its impedance from Cauchy data. », Inverse Problems vol. 24, $\mathrm{n}^{\circ} 4,2008$.

[16] W. RUNDELL « Recovering an obstacle and a nonlinear conductivity from Cauchy data. », Inverse Problems vol. 24, $\mathrm{n}^{\circ}$ 5, 2008.

[17] U. WeIHS,, F. Krummheuer, V. Dubbel « Zerstorungsfreie Baumdiagnose mittels elektrischer Widerstandstomographie », Jahrbuch der Baumpege, 505- 58, 2001,(Braunschweig : Thalacker Medien). 